\title{
Robotic harvest of a latissimus dorsi flap using a single-port surgical robotic system in breast reconstruction
}

\author{
Oh Young Joo ${ }^{1}$, Seung Yong Song ${ }^{1}$, Dae Hyun Lew ${ }^{1}$, Hyung Seok Park ${ }^{2}$, Dong Won Lee ${ }^{1}$ \\ Departments of ${ }^{1}$ Plastic and Reconstructive Surgery and ${ }^{2}$ Surgery, Yonsei University College of Medicine, Seoul, Korea
}

Robot-assisted surgery is evolving to incorporate a higher number of minimally invasive techniques. There is a growing interest in robotic breast reconstruction that uses autologous tissue. Since a traditional latissimus dorsi (LD) flap leads to a long donor scar, which can be an unpleasant burden to patients, there have been many attempts to decrease the scar length using minimally invasive approaches. This study presents the case of a patient who underwent a robot-assisted nipple-sparing mastectomy followed by immediate breast reconstruction with an LD flap using a single-port robotic surgery system. With the assistance of a single-port robot, a simple docking process using a short and less visible incision is possible. Compared to multiport surgery systems, single-port robots can reduce the possibility of collision between robotic arms and provide a clear view of the medial border of the LD where the curvature of the back restricts the visual field. We recommend the use of single-port robots as a minimally invasive approach for harvesting LD flaps.

Keywords Mammaplasty / Robotic surgical procedures / Single port / Latissimus dorsi flap / Breast neoplasms
Correspondence: Dong Won Lee Department of Plastic and Reconstructive Surgery, Yonsei University College of Medicine, 50-1 Yonsei-ro, Seodaemun-gu, Seoul 03722 , Korea

Tel: +82-2-2228-2215

Fax: +82-2-393-6947

E-mail:xyphoss@yuhs.ac

\section{果}

This article was presented at the 10th Global Breast Cancer Conference (GBCC) on April 8-10, 2021, in Seoul, Korea.

The authors thank Medical Illustration \& Design, part of the Medical Research Support Services of Yonsei University College of Medicine, for all artistic support related to this work.

Received: April 29, 2021 • Revised: June 15, 2021 - Accepted: June 16, 2021

pISSN: 2234-6163 • elSSN: 2234-6171 • https://doi.org/10.5999/aps.2021.00710 • Arch Plast Surg 2021;48:577-582

\section{INTRODUCTION}

The field of robotic surgery is currently undergoing vast improvements in its use of minimally invasive techniques due to new developments in ergonomic robotic arms and other instruments [1-3]. Breast reconstruction techniques have improved over time to accommodate the preferences of patients for more natural and aesthetically pleasing results. Consequently, robotic breast reconstruction techniques using autologous tissue have generated great interest. Latissimus dorsi (LD) flaps are among the most common methods for autologous breast reconstruc- tion surgery. Traditionally, harvesting an LD flap requires a long incision that leaves an unsightly scar. Thus, many attempts have been made to reduce postoperative scars using minimally invasive techniques. Shortly after the first study on laparoscopic LD flap harvest was published in the 1990s [4,5], Selber et al. [1] published the first study on robotic LD flap harvest, after which Chung et al. [6] published a study on a different technique involving gasless robotic $\mathrm{LD}$ flap harvest.

To harvest an LD flap with a short incision, a minimally invasive approach is required. However, reaching the medial border of the LD with a camera scope and dissectors is a challenge. 
Since the muscle curves posteromedially following the curvature of the back, the rigid chest wall restricts the manipulation of rigid laparoscopic instruments. This limitation can be overcome with flexible robotic arms. A new single-port robotic system, the da Vinci SP surgical system (Intuitive Surgical), provides flexible camera motion that covers the entire surgical field of the LD flap. It also features articulated robotic arms and a wide range of possible motions to minimize collisions between robotic arms [7]. Given these features, a single-port robotic system can be a feasible option with a flatter learning curve that yields reproducible results for harvesting $\mathrm{LD}$ flaps in breast reconstruction surgery.

\section{IDEA}

\section{Patient selection}

In conventional breast reconstruction using LD flaps for moderate-sized to large breasts, an implant is typically used since an LD flap does not have enough volume on its own. Robotically harvested LD flaps are even more limited in volume since a skin flap is no longer present [8]. A robotic approach to harvesting LD flaps should be considered for situations when only muscle is required without a skin flap. We suggest the following indications for robotic harvest of LD flaps in breast reconstruction: (1) breast reconstruction using an LD flap with fat grafts for small breasts after nipple-sparing mastectomy; (2) oncoplastic surgery using an LD flap after breast-conserving surgery; (3) delayed immediate reconstruction with implants in an irradiated breast [9]; (4) implant coverage instead of acellular dermal matrix for implant-based reconstruction; and (5) chest-wall reconstruction for patients with Poland syndrome. In this study, we introduce a technique for breast reconstruction using $\mathrm{LD}$ flaps with fat grafts on small breasts using a single-port surgical robot system following robot-assisted nipple-sparing mastectomy.

\section{Patient and oncologic surgery}

A 40-year-old patient with no remarkable medical or family history who had been diagnosed with left breast cancer was referred to our breast cancer clinic. A tumor was located in the lower central area of the patient's left breast. Breast tomosynthesis revealed a mass with a density of $31 \mathrm{~mm}$ in the lower inner-to-central area of the left breast. Ultrasonography of the breast demonstrated a hypoechoic area spanning $24 \mathrm{~mm}$ in the 6-o'clock direction, 1 $\mathrm{cm}$ from the nipple. A core needle biopsy of this lesion revealed invasive ductal carcinoma. Additionally, magnetic resonance imaging (MRI) of the breast indicated low suspicious enhancing lesions of approximately $9 \mathrm{~mm}$ and $7 \mathrm{~mm}$ in the medio-central and upper central parts of the left breast, respectively. A core needle biopsy was conducted on the low suspicious lesion found in the 12-o'clock direction and revealed a ductal carcinoma in situ. Several small lymph nodes were noted in the left axilla on the breast MRI. The patient's body mass index was 20.44 $\mathrm{kg} / \mathrm{m}^{2}$, and the volume of each breast was measured using a three-dimensional scanner [10], with estimated measurements of $163 \mathrm{cc}$ (right side) and $189 \mathrm{cc}$ (left side). Due to the patient's small breast size and her desire to minimize surgical scars, we proposed robot-assisted nipple-sparing mastectomy followed by immediate breast reconstruction using a robotically harvested LD flap.

The oncologic surgeons made a $4.5 \mathrm{~cm}$-long longitudinal incision in the mid-axillary line and manually performed sentinel lymph node biopsy. After adequate space was prepared, the da Vinci SP surgical system (Intuitive Surgical, Sunnyvale, CA, USA) was docked, and the robot-assisted nipple-sparing mastectomy was performed [11].

\section{Dissection of the pedicle}

After mastectomy, the anterior border of the LD muscle was visible through the short linear incision made by the oncologic surgeon. The thoracodorsal pedicle, which passes from the axilla along the anterior border of the LD muscle and enters into the deep surface of the LD muscle, was identified. The thoracodorsal nerve, which lies next to the vessel, was ligated to prevent unnecessary twitching of the reconstructed breast. Some plastic surgeons prefer not to ligate the nerve to prevent atrophic changes in the transferred LD muscle.

\section{Patient position and design}

After pedicle dissection, the patient was placed in the lateral decubitus position with the arm positioned below shoulder level to prevent collision with the robotic arm. The operative field was draped so that the ipsilateral breast and back were sufficiently exposed. Once the patient was prepared, important anatomical landmarks were marked: the posterior axillary line, posterior midline, and inferior scapula tip. Then, the outline of the LD muscle to be harvested was drawn (Fig. 1). We recommend drawing the lateral margin slightly medial to the posterior axillary line to enable the robotic arms to make more relaxed motions. A maximum distance of $24 \mathrm{~cm}$ from the port is suggested for the harvest margin of the $\mathrm{LD}$ muscle since that is the maximum reach of the robotic arms. Although the reach of the robotic arms is limited, most of the LD muscle can still be harvested.

\section{Robotic docking}

The establishment of sufficient working space is essential before placing the single-port device. Using a long-tip electrocautery device, the anterior and posterior aspects of the LD muscle were 

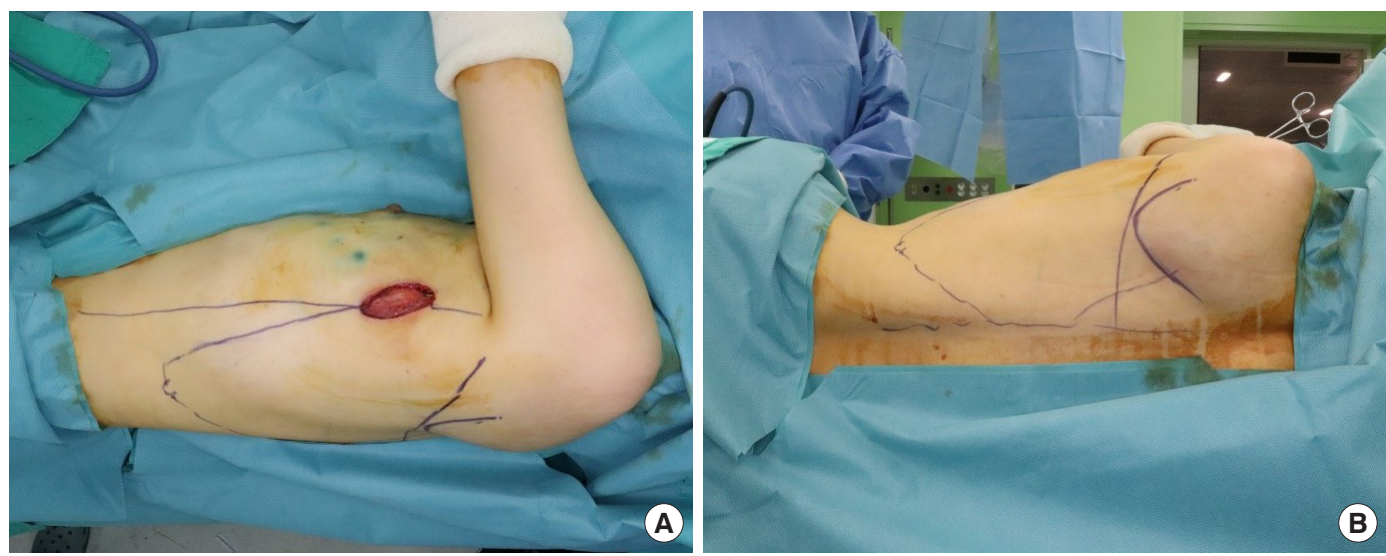

Fig. 1. Significant anatomical landmarks and latissimus dorsi muscle to be harvested. (A) Lateral and (B) posterior views.
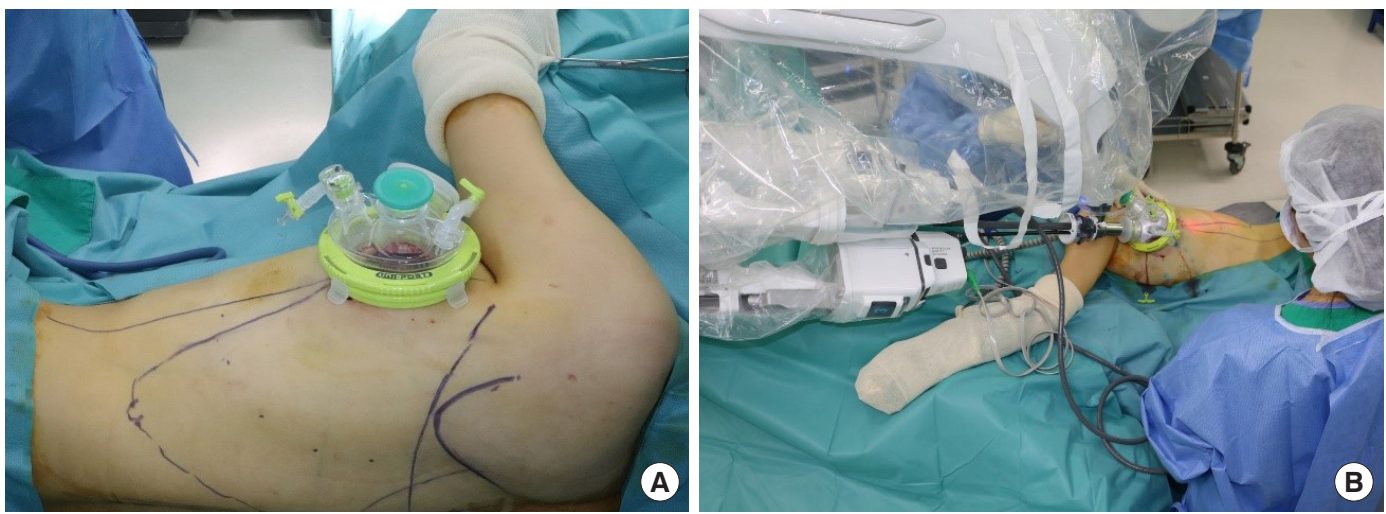

Fig. 2. Docking process. (A) Attachment of the Uniport single-port device and (B) robotic docking.

dissected to create a $10-$ to $15-\mathrm{cm}$ space around the incision. Without additional incisions, a single-port device (Uniport; Dalim, Seoul, Korea) was attached to the axillary incision previously made by the oncologic surgeon (Fig. 2). Once the port device was attached and the port was docked, LD dissection was performed in two steps: first, deep muscle surface dissection (submuscular plane) was performed followed by superficial muscle surface dissection (subcutaneous plane). These dissection steps must be performed in this order since the inflated gas can flow into the superficial space, making it difficult to maintain a working space while dissecting the deep surface [1]. During the first step, the tip of the port was placed under the LD muscle, and carbon dioxide insufflation pressure was maintained at $8 \mathrm{mmHg}$.

After these steps, we docked the robotic arms in the ports. The axis of the arms was aligned toward the patient's T12 vertebra from the axillary incision. The robotic instruments used were the da Vinci SP Cadiere grasping forceps (Intuitive Surgical) for the first instrument drive and the da Vinci SP monopolar curved scissors (Intuitive Surgical) for the second instrument drive. It is important to dock the camera on the upper side of the port to overcome visual field limitations when operating on the medial side where the chest wall is curved.

\section{Robotic harvest of the LD muscle}

Submuscular dissection in the medial and inferior directions was carefully performed. Perforating vessels were coagulated using monopolar curved scissors. Dissection continued until the borders of the design were reached, and the muscle or fascia was cut until the fat layer was exposed. After submuscular dissection, the port was repositioned into the subcutaneous plane. The same dissection procedure was repeated on a different plane, and the muscle border was separated (Fig. 3). The robot was undocked, and the single-port device was detached. Usually, an attachment remains around the teres major muscle and inferior scapula tip. This can be easily detached using long-tip electrocautery and a lighted retractor. The separated LD muscle was transferred into the breast pocket. The overall surgical procedure is shown in Supplemental Video 1.

\section{Fat graft and flap insetting}

Lastly, the patient was moved to a supine position with ipsilater- 

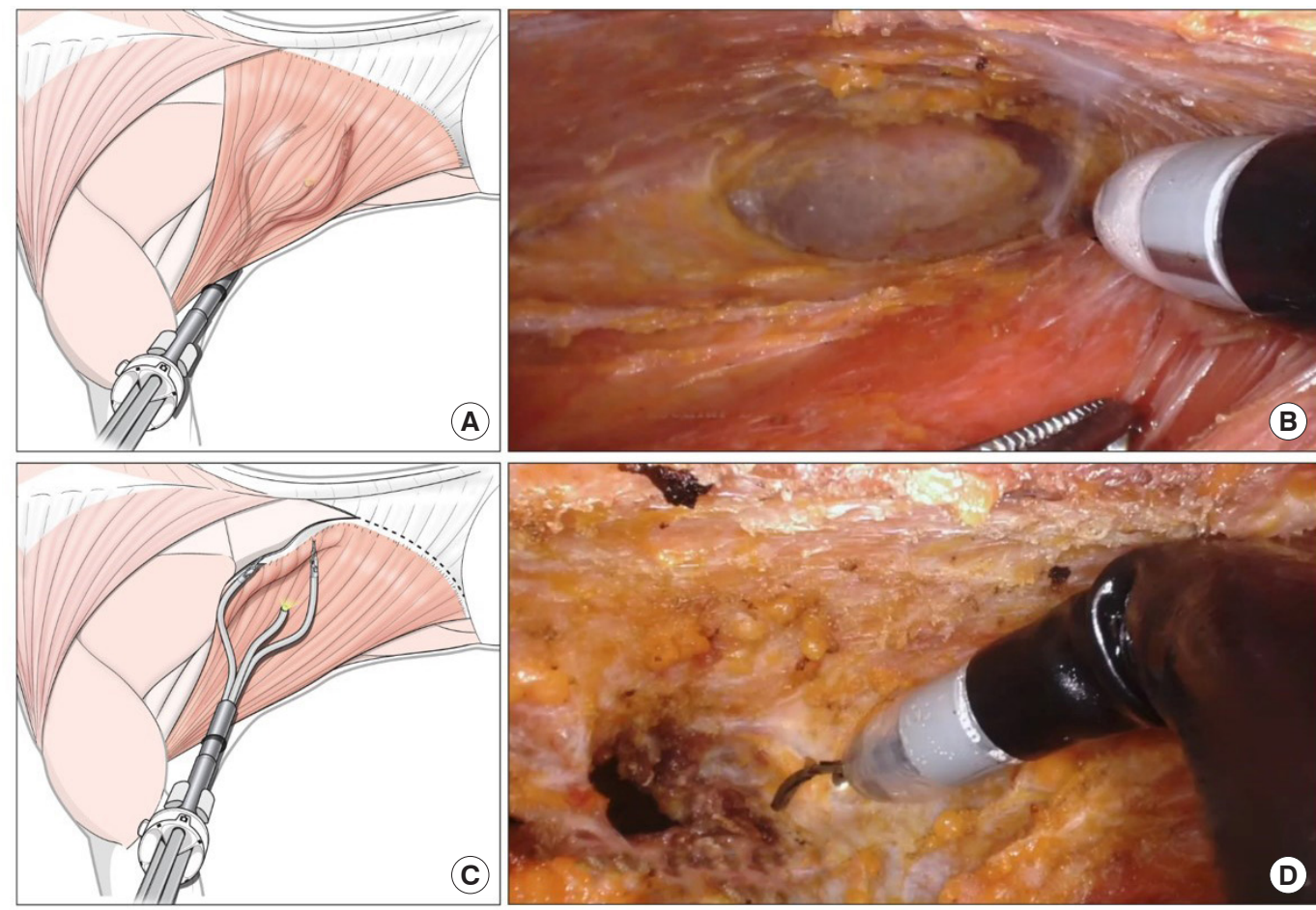

Fig. 3. Complete procedure for harvesting the latissimus dorsi muscle. (A, B) Animated and pictorial image of submuscular robotic dissection. (C, D) Animated and pictorial image of subcutaneous robotic dissection.

al arm abduction. Even for patients with small breasts, it is difficult to fill the total breast volume with one harvested muscle. Thus, fat grafting or an additional breast implant is needed to balance the breasts. Autologous fat, which is commonly harvested from the abdomen or thigh, was grafted into the LD muscle and into the pectoralis major muscle to augment the upper pole. The LD muscle flap was fixed using pull-out sutures. Overall, this method can restore breast volume up to 150-200 cc.

\section{Operation summary}

The total operation time for the robot-assisted nipple-sparing mastectomy was 192 minutes, and the total time for the robotassisted breast reconstruction was 328 minutes, which included the time used for specimen retrieval, irrigation, bleeding control, and preparation of a new operative field. Docking for the robotic flap harvest took 15 minutes. The console time was $100 \mathrm{~min}$ utes. The weight of the retrieved specimen was $172 \mathrm{~g}$. A total of $250 \mathrm{cc}$ of autologous fat was harvested and decanted from both thigh areas. Next, $100 \mathrm{cc}$ of fat was grafted to the LD muscle flap, and $48 \mathrm{cc}$ of fat was grafted to the ipsilateral pectoralis major muscle. There were no major or minor complications such as conversion to open surgery, hematoma, or nipple or skin necrosis. A small seroma ( $<20 \mathrm{cc}$ ) was noted after the drain was removed, but it healed during outpatient care after repeated aspiration.
The final pathology evaluation revealed invasive ductal carcinoma. The maximum diameters of the invasive carcinoma and in situ carcinoma were $1.9 \mathrm{~cm}$ and $4.1 \mathrm{~cm}$, respectively. Expression of the estrogen and progesterone receptors was observed in $90 \%$ and $80 \%$ of the tumor cells, respectively. Tissue evaluations were unclear in terms of human epidermal growth factor receptor 2 expression. Ki-67 expression was evaluated using Roche iScan (Ventana Digital Pathology, Inc, Sunnyvale, CA, USA), and expression of the Ki-67 (30-9) antibody was observed in $15.18 \%$ of the tumor cells. Twelve lymph nodes were harvested. Metastatic carcinoma (maximum diameter: $5 \mathrm{~mm}$ ) was identified in one of the 12 lymph nodes, and perinodal soft tissue extension was not noted. The patient had no postoperative complications and was discharged on postoperative day 6. MammaPrint (Agendia, Inc., Amsterdam, the Netherlands) revealed that the patient had a high genomic and clinical risk of recurrence. She received adjuvant chemotherapy followed by radiotherapy and was prescribed an estrogen receptor antagonist.

The patient was satisfied with the results of the procedure, especially with the inconspicuous scar. A preoperative and postoperative photo of the patient is shown in Figs. 4, 5. The patient completed a survey using the BREAST-Q reconstruction module scale [12]. The results were measured on a scale of $0-100 \mathrm{ac}-$ cording to the BREAST-Q protocol, with a higher value indicating a more favorable outcome. The patient's overall satisfaction 

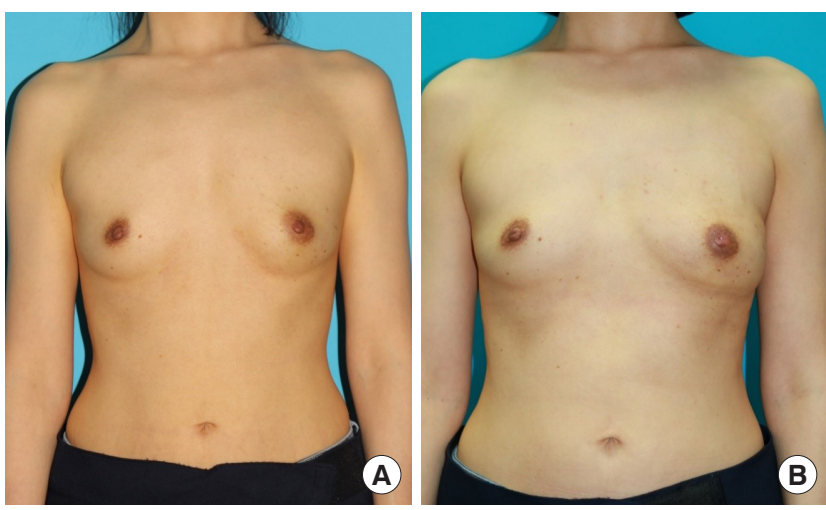

Fig. 4. (A) Preoperative and (B) postoperative (2 months after surgery) frontal views.

score was 67 , which was higher than the average score (55.12) of 152 other patients who underwent breast reconstruction around the same time. The patient's score for her back was 90 .

\section{DISCUSSION}

Robot-assisted breast reconstruction using an LD flap combined with a robot-assisted nipple-sparing mastectomy is an excellent surgical option for yielding positive aesthetic outcomes since only one short longitudinal incision is needed for the whole operation. Traditional breast reconstruction using an LD flap requires a 15- to $45 \mathrm{~cm}$-long dorsal incision. Even though the scar can be hidden under a bra strap, the long incision scar at the donor site can pose an aesthetic burden for patients. Robotassisted reconstruction can avoid such scar formation and improve quality of life.

The simplicity of the docking process, the feasibility of securing a clear view of the LD medial border, and the ease of manipulation using ergonomic robotic arms are the main advantages of the single-port robotic system. No additional incisions are needed after the mastectomy is performed by the general surgeon. In addition, the articulating instruments help to avoid collisions between robotic arms. Moreover, in the authors' experiences, compared to laparoscopic or other multiport robotic systems, the da Vinci SP system has a much shorter learning curve.

With public interest in robotic surgery growing, robotic surgery has only just begun to be applied to breast reconstruction. Limitations remain for this particular surgical application, such as the high price and costly maintenance of the da Vinci SP system and the fact that robotic mastectomy and breast reconstruction have not been approved by the Food and Drug Administration. To overcome these barriers, standardized procedures must be established and further studies should be conducted to assess patients' 30-day safety after the procedure and long-term com-
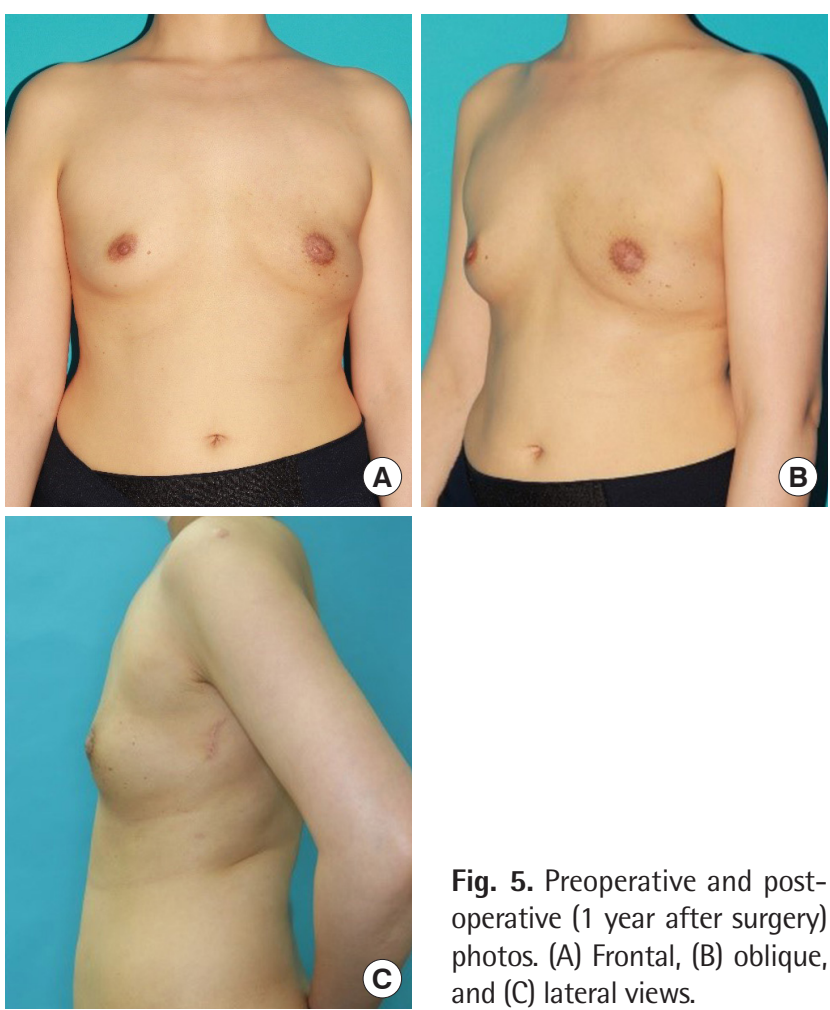

Fig. 5. Preoperative and postoperative (1 year after surgery) photos. (A) Frontal, (B) oblique, and $(C)$ lateral views.

plication risks [13].

Additionally, when using a multiport robotic system, the robotic arms and camera are parallel and can conflict with one another at the medial and lateral extremes of the dissection. A single-port robotic system overcomes this limitation and minimizes collisions between robotic arms. Moreover, the flexible camera helps to observe all structures without blind spots, especially in medial areas where the curvature of the back can restrict the visual field.

The advantages of the new surgical system include a simple docking process, flexible camera motions, and minimization of collisions between robotic arms. The surgical technique outlined in this study involving immediate breast reconstruction with an LD flap using the da Vinci SP surgical system seems to be a feasible and safe option for minimally invasive breast reconstruction procedures.

\section{NOTES}

\section{Conflict of interest}

Seung Yong Song is editorial board member of the journal but was not involved in the peer reviewer selection, evaluation, or decision process of this article. No other potential conflicts of interest relevant to this article were reported. 


\section{Ethical approval}

The study was approved by the Institutional Review Board of Severance Hospital (IRB No. 1-2021-0017) and performed in accordance with the principles of the Declaration of Helsinki. The IRB waived the requirement for informed consent and approved the design of this retrospective study.

\section{Patient consent}

The patients provided written informed consent for the publication and the use of their images.

\section{Author contribution}

Conceptualization: DW Lee. Data curation: OY Joo, HS Park. Formal analysis: OY Joo. Methodology: SY Song, DH Lew, DW Lee. Project administration: DW Lee. Writing - original draft: OY Joo. Writing - review \& editing: DW Lee. All authors read and approved the final manuscript.

\section{ORCID}

Oh Young Joo https://orcid.org/0000-0001-5736-4671

Seung Yong Song https://orcid.org/0000-0002-3145-7463

Dae Hyun Lew https://orcid.org/0000-0002-2625-5664

Hyung Seok Park https://orcid.org/0000-0001-5322-6036

Dong Won Lee https://orcid.org/0000-0003-0046-3139

\section{Supplementary material}

Supplemental Video 1. A video showing the entire reconstruction operation procedure. Supplemental data can be found at: https://doi.org/10.5999/aps.2021.00710.v001

\section{REFERENCES}

1. Selber JC, Baumann DP, Holsinger FC. Robotic latissimus dorsi muscle harvest: a case series. Plast Reconstr Surg 2012;129:1305-12.

2. Agarwal DK, Sharma V, Toussi A, et al. Initial experience with da Vinci single-port robot-assisted radical prostatecto- mies. Eur Urol 2020;77:373-9.

3. Ahn SJ, Song SY, Park HS, et al. Early experiences with robot-assisted prosthetic breast reconstruction. Arch Plast Surg 2019;46:79-83.

4. Fine NA, Orgill DP, Pribaz JJ. Early clinical experience in endoscopic-assisted muscle flap harvest. Ann Plast Surg 1994;33:465-9.

5. Bass LS, Karp NS, Benacquista T, et al. Endoscopic harvest of the rectus abdominis free flap: balloon dissection in the fascial plane. Ann Plast Surg 1995;34:274-9.

6. Chung JH, You HJ, Kim HS, et al. A novel technique for robot assisted latissimus dorsi flap harvest. J Plast Reconstr Aesthet Surg 2015;68:966-72.

7. Lee J, Park HS, Lee H, et al. Axillary lymph node dissection using a robotic surgical system: initial experience. J Surg Oncol 2020;122:1252-6.

8. Chang HP, Fan KL, Song SY, et al. The traditional versus endoscopic-assisted latissimus dorsi harvest in oncoplastic surgery: a long term comparison of breast volume, aesthetics, and donor site outcomes. Asian J Surg 2020;43:1165-71.

9. Clemens MW, Kronowitz S, Selber JC. Robotic-assisted latissimus dorsi harvest in delayed-immediate breast reconstruction. Semin Plast Surg 2014;28:20-5.

10. Lee WY, Kim MJ, Lew DH, et al. Three-dimensional surface imaging is an effective tool for measuring breast volume: a validation study. Arch Plast Surg 2016;43:430-7.

11. Park HS, Lee J, Lee DW, et al. Robot-assisted nipple-sparing mastectomy with immediate breast reconstruction: an initial experience. Sci Rep 2019;9:15669.

12. Pusic A, Klassen A, Cano S. Breast-Q version 2.0@ reconstruction module pre- and postoperative scales. Memorial Sloan Kettering Cancer Center and the University of British Columbia; 2017.

13. Donnely E, Griffin MF, Butler PE. Robotic surgery: a novel approach for breast surgery and reconstruction. Plast Reconstr Surg Glob Open 2020;8:e2578. 